\title{
Exercise-induced albuminuria is associated with perivascular renal sinus fat in individuals at increased risk of type 2 diabetes
}

\author{
R. Wagner • J. Machann • R. Lehmann • K. Rittig • \\ F. Schick • J. Lenhart $\cdot$ F. Artunc $\cdot$ K. Linder • \\ C. D. Claussen • E. Schleicher • A. Fritsche • \\ H.-U. Häring • P. Weyrich
}

Received: 27 December 2011 / Accepted: 15 March 2012/Published online: 24 April 2012

(C) Springer-Verlag 2012

\begin{abstract}
Aims/hypothesis Microalbuminuria represents an established surrogate marker of early diabetic nephropathy and glomerular microangiopathy. Increasing evidence is emerging of a role of perivascular adipose tissue (PVAT) as an important link between obesity, insulin resistance and both macro- and microangiopathy. It is not known whether perivascular renal sinus fat (RSF) has an impact on microalbuminuria in the prediabetic stage. We investigated whether RSF quantified by MRI is associated with microalbuminuria before or after exercise.
\end{abstract}

R. Wagner $\cdot$ R. Lehmann $\cdot$ K. Rittig $\cdot$ F. Artunc $\cdot$ K. Linder $\cdot$ E. Schleicher $\cdot$ A. Fritsche $\cdot$ H.-U. Häring $\cdot$ P. Weyrich $(\triangle)$ Department of Internal Medicine, Division of Endocrinology, Diabetology, Angiology, Nephrology and Clinical Chemistry, Eberhard Karls University Tübingen,

Otfried-Müller-Str 10,

72076 Tübingen, Germany

e-mail: peter.weyrich@med.uni-tuebingen.de

J. Machann $\cdot$ F. Schick $\cdot$ J. Lenhart

Section on Experimental Radiology,

Eberhard Karls University Tübingen,

Tübingen, Germany

R. Lehmann • E. Schleicher · A. Fritsche $\cdot$ H.-U. Häring Institute for Diabetes Research and Metabolic Diseases of the Helmholtz Centre Munich at the University of Tübingen (Paul Langerhans Institute Tübingen),

Tübingen, Germany

C. D. Claussen

Department of Diagnostic and Interventional Radiology,

Eberhard Karls University Tübingen,

Tübingen, Germany
Methods Non-diabetic individuals at increased risk of type 2 diabetes were recruited into the Tübingen Lifestyle Intervention Program (TULIP); 146 participants took part in the analysis. RSF was measured in axial MRI sections at the level of the renal artery. Urine was collected before and after exercise stress testing.

Results Participants (age $47 \pm 12$ years; mean \pm SD) reached a mean exercise load of $176 \pm 49 \mathrm{~W}$, with a mean arterial peak pressure (MAPP) of $112 \pm 14 \mathrm{mmHg}$. After adjusting for sex, age, visceral adipose tissue (VAT) and MAPP during exercise, RSF was significantly associated with postexercise albumin/ creatinine ratio (ACR; $p=0.006$ ). No association between RSF and baseline BP could be observed after adjusting for confounders $(p=0.26)$, and there was no association between RSF and baseline ACR either $(p=0.2)$.

Conclusions RSF is associated with exercise-induced albuminuria independently of sex, age, VAT and MAPP in a nondiabetic cohort at diabetic risk. We conclude that PVAT in the renal sinus may play a role in the pathogenesis of microalbuminuria.

Keywords Diabetic nephropathy $\cdot$ Perivascular fat $\cdot$ Renal sinus fat $\cdot$ Type 2 diabetes
Abbreviations
ACR Albumin/creatinine ratio
MAPP Mean arterial peak pressure
PVAT Perivascular adipose tissue
RSF Renal sinus fat
TULIP Tübingen Lifestyle Intervention Program
VAT Visceral adipose tissue 


\section{Introduction}

Despite the remarkable efforts that have gone into researching the pathogenesis of diabetic nephropathy, no reliable predictors have been found. Obesity is associated with diabetic nephropathy risk independently of glycaemic control and hypertension. While liver fat [1] and perivascular adipose tissue (PVAT) [2] represent established metabolic determinants, data on the role of different adipose tissue compartments in the development of diabetic nephropathy are still lacking. Renal sinus fat (RSF) constitutes the renal PVAT compartment surrounding the renal artery, renal vein, lymphatic vessels and proximal ureteral portion. PVAT secretes a variety of paracrine factors interacting with immune cells, vascular endothelium and smooth muscle cells [3]. RSF mass is associated with hypertension in diabetes and chronic kidney disease $[4,5]$.

Given these data, we hypothesised that RSF may play a role in the pathogenesis of diabetic nephropathy and may therefore be associated with microalbuminuria. Several studies have shown that exercise unmasks albuminuria in normoalbuminuric diabetic individuals, assuming that exerciseinduced albuminuria may be the earliest sign of diabetic nephropathy. Exercise leads to albuminuria by both modulating glomerular passage and tubular reuptake of albumin [6]. We investigated whether pre- and/or postexercise urinary albumin/creatinine ratio (ACR) is associated with RSF in individuals at diabetic risk.

\section{Methods}

Participants Participants were recruited from the Tübingen Lifestyle Intervention Program (TULIP), which investigates possible predictors of successful lifestyle intervention. Individuals at increased diabetic risk (family history of type 2 diabetes, BMI $>27 \mathrm{~kg} / \mathrm{m}^{2}$, impaired glucose tolerance or previous gestational diabetes) were recruited from southern Germany. The study protocol was approved by the local ethics committee in accordance with the Helsinki Declaration. All participants gave their written informed consent.

Based upon the availability of urine specimens from the TULIP baseline examination, 176 TULIP participants were selected for RSF quantification. Exclusion of participants with a single kidney $(n=1)$, pelvic kidneys $(n=2)$ or insufficient MRI datasets $(n=5)$ resulted in 168 participants. An exercise test was conducted in 162 participants, of whom 13 were not able to provide postexercise urine within $30 \mathrm{~min}$ after cycling. Thus, postexercise ACR was available for 149 participants. For three of these participants, BP data during exercising were incomplete, resulting in a final study cohort of 146 individuals.

Exercise test and laboratory measurements The participants provided morning spot urine after an overnight fast. An
OGTT was performed using a bedside glucose analyser (YSI, Yellow Springs, OH, USA). The participants subsequently underwent an exercise test until exhaustion on a treadmill bicycle (Ergometrics $800 \mathrm{~S}$; ergoline, Bitz, Germany). BP was measured with a sphygmomanometer (Prakticus II; Friedrich Bosch, Bisingen, Germany) before and every 2 min during exercise. Spot urine was collected within $30 \mathrm{~min}$. Urinary albumin was measured nephelometrically (BN ProSpec Nephelometer) and creatinine quantified enzymatically (ADVIA 1800 Clinical Chemistry System; both Siemens Healthcare Diagnostics, Eschborn, Germany).

Measurement of adipose tissue MRI (1.5 T whole-body imager, Magnetom Sonata; Siemens Healthcare Diagnostics) was performed in the early morning as described previously [7]. Axial images were recorded applying a T1-weighted fast spin-echo technique: echo time $12 \mathrm{~ms}$, repetition time $490 \mathrm{~ms}$, turbo factor 7, slice thickness $10 \mathrm{~mm}$, gap between slices $10 \mathrm{~mm}$, field of view $480-560 \mathrm{~mm}$. For determination of RSF, the slice which best depicts the entry of the renal artery into the corresponding kidney was selected for each kidney. Two regions of interest were outlined for both kidneys as shown in Fig. 1. Region A (Fig. 1a, dashed line, blue arrow) was chosen for determination of the total kidney volume at this level using a planimetric approach. Region B (continuous line, white arrow) was selected for subsequent measurement of adipose tissue within the renal sinus. In addition, visceral adipose tissue (VAT) and subcutaneous adipose tissue at the umbilical level were quantified [7].

In order to exclude a systematic bias in RSF measurement (different kidney sizes, etc.), the ratio of renal sinus adipose tissue to total kidney size was calculated using the arithmetical mean (left/right kidney RSF) for each participant. To prove that the selected MRI slices at the level of entry of the renal artery represented the largest renal sinus diameters, control measurements on cranially and caudally neighbouring slices were carried out. Ten participants were randomly selected in every BMI tertile. Cranially adjacent sections demonstrated $4.1 \pm 3.2 \%$ points $(p<0.0001$, paired two-tailed $t$ test) lower RSF content than the original planes. Caudally adjacent section planes amounted to $3.2 \pm 2.7 \%$ points lower RSF content $(p<0.0001)$. Since both upper and lower adjacent sections contain significantly less RSF, the selected planes at the entry of the renal artery depict the renal sinuses at their largest diameter and were accepted as a representative surrogate variable for total RSF.

Calculations $\mathrm{BP}$ is expressed as mean arterial BP calculated as $2 / 3 \times$ diastolic $\mathrm{BP}+1 / 3 \times$ systolic $\mathrm{BP}$. RSF is expressed as a percentage (see above). Glomerular filtration rate was estimated using the Chronic Kidney Disease Epidemiology Collaboration (CKD-EPI) equation. Data are given as mean $\pm \mathrm{SD}$. Variables with skewed distributions were logarithmically 

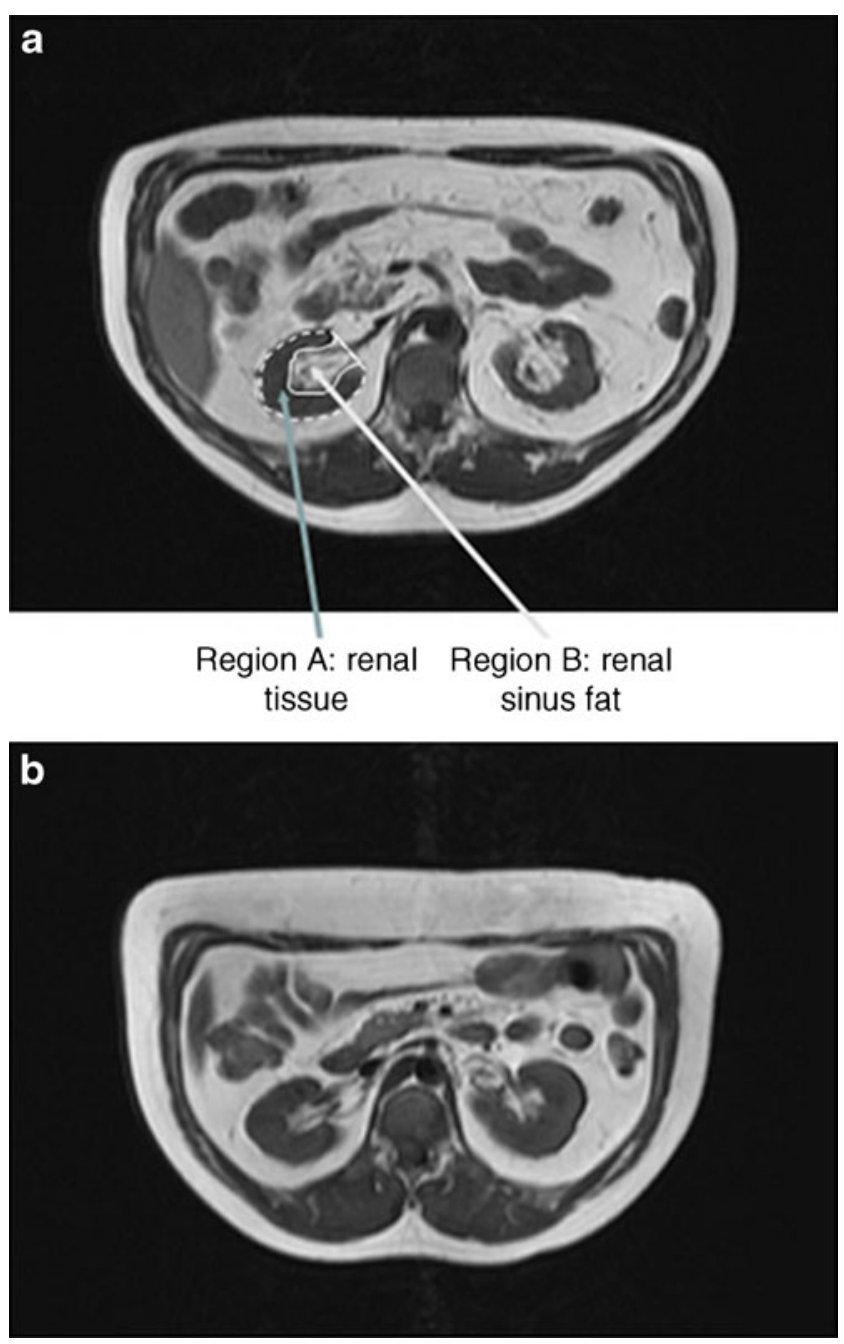

Fig. 1 Axial T1-weighted magnetic resonance images taken at the level of entry of the renal arteries into the renal sinus in two subjects with comparable BMI. a Male volunteer (BMI $32.3 \mathrm{~kg} / \mathrm{m}^{2}$ ) with a high amount of PVAT in the renal sinus (RSF). Manually drawn regions of interest for quantification of total kidney area (region A, dashed line, blue arrow) and adipose tissue in the renal sinus (region B, continuous line, white arrow) are drawn in the right kidney. b Male volunteer (BMI $33.5 \mathrm{~kg} / \mathrm{m}^{2}$ ) with a low amount of RSF on both sides. RSF was calculated as the arithmetical mean of $\mathrm{B} /(\mathrm{A}+\mathrm{B})$ left and right

transformed to approximate normal distributions. Associations were assessed by multiple linear regression models using least-squares estimations. Effect sizes are given as standardised beta coefficients ( $\left.\beta_{\mathrm{st}}\right)$ with $95 \% \mathrm{CI} ; p<0.05$ was considered statistically significant. Statistical calculations were performed using JMP 8.0 (SAS, Cary, NC, USA).

\section{Results}

RSF amounted to $6.9 \pm 5.1 \%$ of kidney areas. RSF was associated with sex, being significantly higher in male (9.7 $\pm 5.7 \%)$ than in female participants $(5.0 \pm 3.7 \% ; p<0.001)$. RSF was positively associated with age $\left(\beta_{\text {st }} 0.44 ; 95 \%\right.$ CI $0.29,0.59)$, BMI $\left(\beta_{\mathrm{st}} 0.3 ; 95 \%\right.$ CI $\left.0.15,0.46\right)$ and VAT $\left(\beta_{\mathrm{st}}\right.$ $0.64 ; 95 \%$ CI $0.52,0.77$; all $p<0.001)$. In a multiple linear regression model with sex $\left(\beta_{\mathrm{st}} 0.27 ; 95 \%\right.$ CI $0.13,0.40$ for males), age $\left(\beta_{\mathrm{st}} 0.44 ; 95 \% \mathrm{CI} 0.31,0.57\right)$ and $\mathrm{BMI}\left(\beta_{\mathrm{st}} 0.28\right.$; $95 \%$ CI $0.15,0.41)$ as explanatory variables, all variables were significantly associated with RSF $(p<0.001)$, but further adjustment for VAT $\left(\beta_{\mathrm{st}} 0.55 ; 95 \%\right.$ CI $0.34,0.76 ; p<$ $0.001)$ rendered the influence of BMI insignificant $\left(\beta_{\mathrm{st}}-0.03\right.$; $95 \% \mathrm{CI}-0.20,0.14 ; p=0.68)$. RSF showed no association with subcutaneous adipose tissue $\left(\beta_{\mathrm{st}} 0.09 ; 95 \% \mathrm{CI}-0.07,0.26 ; p=\right.$ $0.28)$. Smoking showed a trend for an association with postexercise ACR $\left(\beta_{\mathrm{st}} 0.14,95 \% \mathrm{CI}-0.02,0.30 ; p=0.08\right)$ after adjusting for sex, age and BMI, but not with pre-exercise $\operatorname{ACR}\left(\beta_{\mathrm{st}}-0.09 ; 95 \% \mathrm{CI}-0.25,0.07 ; p=0.35\right)$. Use of antihypertensive or cholesterol-lowering agents was not associated with pre- $\left(\beta_{\mathrm{st}}-0.02 ; 95 \% \mathrm{CI}-0.20,0.16 ; \beta_{\mathrm{st}} 0.13 ; 95 \% \mathrm{CI}\right.$ $-0.04,0.30)$ or postexercise ACR $\left(\beta_{\mathrm{st}}-0.04 ; 95 \% \mathrm{CI}-0.22\right.$, $0.14 ; \beta_{\text {st }} 0.03 ; 95 \%$ CI $\left.-0.14,0.20 ; p>0.77\right)$ in identical models.

Estimated glomerular filtration rate was not associated with RSF after adjustment for age and sex $\left(\beta_{\mathrm{st}}-0.02 ; 95 \%\right.$ CI $-0.11,0.07 ; p=0.6) \mathrm{with} /$ without further adjustment for VAT $\left(\beta_{\text {st }}-0.04 ; 95 \%\right.$ CI $\left.-0.14,0.06 ; p=0.46\right)$.

RSF was not associated with ACR quantified in the morning spot urine (pre-exercise microalbuminuria) and adjusted for sex, age, VAT and resting BP $\left(\beta_{\mathrm{st}} 0.12 ; 95 \% \mathrm{CI}-0.11\right.$, $0.34 ; p=0.19)$. However, RSF was positively associated with postexercise ACR after adjustment for sex, age, VAT and mean arterial peak pressure (MAPP) during exercise (Table 1). RSF maintained its significant association with postexercise ACR $\left(\beta_{\text {st }} 0.30 ; 95 \%\right.$ CI $\left.0.09,0.51 ; p=0.005\right)$ even after further adjustment for peak exercise workload. Running association models between RSF and postexercise ACR adjusted for smoking $\left(\beta_{\text {st }} 0.05 ; 95 \%\right.$ CI $-0.12,0.21 ; p=0.56$ ),

Table 1 Linear regression model depicting the association between postexercise ACR and RSF (independent variable) after adjustment for confounding variables

\begin{tabular}{lrlrll}
\hline $\begin{array}{l}\text { Predictor } \\
\text { variables }\end{array}$ & \multicolumn{1}{l}{$\beta$} & \multicolumn{1}{l}{ SE } & \multicolumn{1}{l}{$\beta_{\text {st }}$} & $95 \%$ CI & $p$ value \\
\hline Sex $(1=$ male $)$ & 0.028 & 0.079 & 0.033 & $-0.15,0.217$ & 0.724 \\
Age & -0.541 & 0.260 & -0.190 & $-0.368,-0.011$ & 0.039 \\
VAT & -0.308 & 0.132 & -0.273 & $-0.502,-0.044$ & 0.021 \\
MAPP & 1.686 & 0.589 & 0.248 & $0.078,0.418$ & 0.005 \\
RSF & 0.254 & 0.091 & 0.303 & $0.091,0.515$ & 0.006 \\
\hline
\end{tabular}

All parametric variables were log-transformed to approximate normal distribution. Effect sizes are given as beta coefficients $(\beta)$, their standard error (SE), standardised beta coefficients $\left(\beta_{\mathrm{st}}\right)$ and confidence intervals for standardised beta coefficients (CI) 
antihypertensive $\left(\beta_{\mathrm{st}}-0.02 ; 95 \% \mathrm{CI}-0.19,0.15 ; p=0.81\right)$ or cholesterol-lowering agents $\left(\beta_{\mathrm{st}} 0.02 ; 95 \% \mathrm{CI}-0.14,0.19 ; p=\right.$ 0.77 ) did not change our main finding regarding the association of RSF with postexercise ACR $\left(\beta_{\mathrm{st}} 0.29 ; 95 \%\right.$ CI 0.07 , $0.51 ; p=0.009 ; \beta_{\mathrm{st}} 0.30 ; 95 \%$ CI $0.09,0.51 ; p=0.006 ; \beta_{\mathrm{st}}$ $0.30 ; 95 \%$ CI $0.09,0.52 ; p=0.006$, respectively).

RSF showed an association with resting BP $\left(\beta_{\mathrm{st}} 0.25 ; 95 \%\right.$ CI $0.09,0.41 ; p=0.003)$, but it exhibited only a trend for an association with MAPP $\left(\beta_{\mathrm{st}} 0.14 ; 95 \% \mathrm{CI}-0.02,0.31 ; p=\right.$ 0.08). RSF did not associate with peak BP after adjustment for sex, age, BMI or VAT $\left(\beta_{\text {st }}-0.03 ; 95 \%\right.$ CI $-0.22,0.16 ; \beta_{\text {st }}$ $-0.09 ; 95 \%$ CI $-0.30,0.11)$, nor after adjustment for peak exercise load ( $\beta_{\mathrm{st}}-0.09 ; 95 \%$ CI $-0.30,0.11$; all $\left.p>0.38\right)$. We decided to adjust our association model between RSF and postexercise ACR for MAPP and not for resting BP, as only MAPP significantly influenced postexercise ACR $\left(\beta_{\text {st }} 0.21\right.$; $95 \%$ CI $0.05,0.37 ; p=0.009)$ compared with resting $\mathrm{BP}\left(\beta_{\mathrm{st}}\right.$ $0.08 ; 95 \%$ CI $-0.08,0.25 ; p=0.33)$.

\section{Discussion}

Several conceivable mechanisms may explain the association between RSF and albuminuria found in this study. PVAT secretes a multitude of cytokines and the secretion profile differs according to the localisation of PVAT in the vascular tree [8]. Thus, RSF representing the PVAT of renal arteries could influence microalbuminuria independently of VAT mass, as shown in our study. Saturated fatty acids increase endoplasmic reticulum stress in insulin-sensitive tissue [9]. Large perivascular triacylglycerol stores could therefore contribute to local inflammation, which is implicated in the pathogenesis of diabetic nephropathy.

PVAT was proposed as a regulator of insulin-induced vasoreactivity, and defective podocyte-specific insulin signalling plays a role in the aetiology of proteinuria through podocyte damage [10]. It seems conceivable that RSF may modulate both insulin-signalling on vasoreactivity and the podocyte cytoskeleton. A mechanical effect of RSF should also be considered, as pressure on the renal veins and lymph vessels can lead to hyperfiltration.

An important point of uncertainty in our study is the testing of exercise-induced albuminuria. Although physiologically plausible and supported by smaller studies [11, 12], exercise-induced albuminuria remains of uncertain prognostic value. Cortical thinning of the kidneys could theoretically confound the analysis, but this is an agerelated phenomenon and age was accounted for in our model. Another limitation might be that the exercise test was carried out immediately after the OGTT. However, this potential confounder was immanent, and $2 \mathrm{~h}$ plasma glucose did not associate with postexercise ACR (data not shown).
Our results can be considered as a further piece in the jigsaw puzzle of understanding human diabetic nephropathy. To validate the potential role of RSF for clinical endpoints, longitudinal studies with long-term follow-up must be undertaken. Identification of individuals at greatest risk of later diabetic nephropathy represents a pivotal future challenge in order to focus nephroprotection on those at highest risk.

Acknowledgements We would like to thank A. Bury, E. Kollmar and B. Horrer (Department of Internal Medicine, Division of Endocrinology, Diabetology, Angiology, Nephrology and Clinical Chemistry, Eberhard Karls University, Tübingen, Germany) for excellent technical assistance.

Funding This study was supported in part by a grant from the German Federal Ministry of Education and Research (BMBF) to the German Centre for Diabetes Research (DZD).

Duality of interest The authors declare that there is no duality of interest associated with this manuscript.

Contribution statement RW contributed to the analysis and interpretation of data, and drafted and revised the manuscript. JM contributed to the acquisition, analysis and interpretation of data, and drafted and revised the manuscript. FS and CDC contributed to the conception and design of the study, and revised the manuscript. KR, JL, FA, KL, $\mathrm{RL}$ and ES contributed to the acquisition of data and revised the manuscript. AF and $\mathrm{H}-\mathrm{UH}$ contributed to the conception and design of the study, the analysis and interpretation of the data, and revised the manuscript. PW contributed to the conception and design of the study, the analysis and interpretation of data, and drafted and revised the manuscript. All authors approved the final version.

\section{References}

1. Stefan N, Häring H-U (2011) The metabolically benign and malignant fatty liver. Diabetes 60:2011-2017

2. Rittig K, Staib K, Machann J et al (2008) Perivascular fatty tissue at the brachial artery is linked to insulin resistance but not to local endothelial dysfunction. Diabetologia 51:2093-2099

3. Meijer RI, Serne EH, Smulders YM, van Hinsbergh VWM, Yudkin JS, Eringa EC (2011) Perivascular adipose tissue and its role in type 2 diabetes and cardiovascular disease. Curr Diab Rep 11:211217

4. Chughtai HL, Morgan TM, Rocco M et al (2010) Renal sinus fat and poor BP control in middle-aged and elderly individuals at risk for cardiovascular events. Hypertension 56:901-906

5. Foster MC, Hwang S-J, Porter SA, Massaro JM, Hoffmann U, Fox CS (2011) Fatty kidney, hypertension, and chronic kidney disease: the Framingham Heart Study. Hypertension 58:784790

6. Poortmans JR, Brauman H, Staroukine M, Verniory A, Decaestecker C, Leclercq R (1988) Indirect evidence of glomerular/tubular mixedtype postexercise proteinuria in healthy humans. Am J Physiol 254: F277-F283 (2 part 2)

7. Machann J, Thamer C, Schnoedt B et al (2005) Standardized assessment of whole body adipose tissue topography by MRI. J Magn Reson Imaging 21:455-462

8. Chatterjee TK, Stoll LL, Denning GM et al (2009) Proinflammatory phenotype of perivascular adipocytes: influence of high-fat feeding. Circ Res 104:541-549 
9. Peter A, Weigert C, Staiger H et al (2009) Individual stearoyl-CoA desaturase 1 expression modulates endoplasmic reticulum stress and inflammation in human myotubes and is associated with skeletal muscle lipid storage and insulin sensitivity in vivo. Diabetes 58:1757-1765

10. Fornoni A (2010) Proteinuria, the podocyte, and insulin resistance. N Engl J Med 363:2068-2069
11. Cesarini PR, Ferreira SR, Vivolo MA, Zanella MT (1996) Different urinary albumin responses to submaximal exercise by normoalbuminuric diabetic children and controls. Braz J Med Biol Res 29:16031610

12. Huttunen NP, Käär ML, Pietiläinen $M$, Vierikko $P$, Reinilä $M$ (1981) Exercise-induced proteinuria in children and adolescents. Scand J Clin Lab Invest 41:583-587 